\title{
Correlation and Path Analysis in Groundnut (Arachis hypogaea L.)
}

\author{
K. John*, M. V. Madhavi Santhoshi and P. Rajasekhar \\ Institute of Frontier Technology, Regional Agricultural Research Station, \\ Tirupati-517502, Andhra Pradesh, India \\ *Corresponding author
}

K e y w o r d s
Pods per plant,
Correlation,
Primary branches,
Relationships,
Components
Article Info
Accepted:
12 November 2019
Available Online:
10 December 2019

\section{A B S T R A C T}

The estimate of genotypic correlation coefficients in general higher than their corresponding phenotypic correlations indicating strong inherent association among the traits. Number of well-filled and mature pods per plant had shown positive correlation with SPAD Chlorophyll meter reading. Shelling per cent registered positive correlation with 100 kernel weight, specific leaf area and pod yield per plant. Pod yield per plant showed positively significant correlation with the traits viz., number of primary branches per plant, number of immature pods per plant and 100 pod weight. These characters can be considered as criteria for selection for higher yield, as these were mutually and directly associated with pod yield. Number of well-filled and mature pod per plant had shown positive and negligible indirect effect on pod yield per plant via dry haulms yield per plant. The highest positive direct effect on pod yield per plant was recorded with number of primary branches per plant followed by sound mature kernel per cent, 100 kernel weight and number of well-filled and mature pods per plant. Hence, a direct selection for this trait would be effective.

\section{Introduction}

Pod yield in groundnut (Arachis hypogaea L.) is a complex and depends upon the interplay of number of components attributes. A clear picture of contribution of each component is the final expression of character would emerge through the study of correlation and causation of path concept revealing different ways in which component attributes influence the complex traits. In order to achieve the goal of increased production by increasing the yield potential of crop, knowledge of direction and magnitude of association between various traits is essential for plant breeders. Understanding the relationships between yield and yield components is of paramount importance for making the best use of these relationships in selection. Correlation is a biometrical approach which brings out the 
intensity of the association between two pairs of characters and provides information on those components that could serve as criteria for selection of candidates in a breeding program. Traits that are positively correlated with yield are considered effective because selection for such traits would result in the simultaneous improvement in yield (Mahalakshmi et al., 2005). The correlation coefficient may be confounded with indirect effect due to common association inherent in trait interrelationships. Path coefficient analysis measures the direct influence of one variable upon another and facilitates the separation of correlation coefficients into components of direct and indirect effects (Dewey and Lu, 1959). Therefore information derived from the correlation coefficients can be augmented by partitioning correlations into direct and indirect effects by path coefficient analysis. Accordingly, the present investigation was aimed to study the association of pod yield and its component traits in groundnut.

\section{Materials and Methods}

The experimental material consisted of eight elite groundnut genotypes and was conducted in a Randomized Block Design with three replications at Regional Agricultural Research Station, Tirupati, Andhra Pradesh under irrigated conditions during rabi 2017-18. All the genotypes were randomized in three replications and were raised in a single row of $3.0 \mathrm{~m}$ length with a spacing of $22.5 \times 10 \mathrm{~cm}$. Five random plants per replication were sampled for recording observations from each genotype per replication and their mean values were used. The experiment was conducted in a red sandy loam soil with a neutral $\mathrm{pH}$, low in organic carbon. Recommended agronomic and plant protection measures were adopted for the conduct of experiment. The data were recorded for thirteen quantitative traits viz., plant height, number of primary branches per plant, number of secondary branches per plant, number of well-filled and mature pods per plant, number of immature pods per plant, dry haulms yield per plant $(\mathrm{g}), 100$ pod weight $(\mathrm{g})$, shelling per cent, 100 kernel weight $(\mathrm{g})$, sound mature kernel per cent, specific leaf area, SAPD Chlorophyll meter reading and pod yield per plant. Genotypic and phenotypic correlation coefficients were calculated among the genotypes using the formulae suggested by Al-Jibouri et al., (1958). Path coefficient analysis was carried out by using phenotypic and genotypic correlation coefficients as per the method suggested by Dewey and Lu (1959).

\section{Correlation}

The results revealed that for all the traits studied, genotypic correlations were higher than their corresponding phenotypic correlations. This may be due to the relative stability of genetic resources as majority of them were subjected to certain amount of selection and are furnished in Table 1.

Plant height shown positive correlation with number of well filled and mature pods per plant $\left(r_{p}=0.2263\right)$, Number of immature pods per plant $\left(r_{p}=0.1882\right)$ and SPAD Chlorophyll meter reading $\left(r_{p}=0.2041\right)$ at phenotypic level and negatively significant for Specific leaf area $\left(r_{p}=-0.1965\right)$ at phenotypic level. Number of primary branches per plant exhibited positive correlation with number of secondary branches per plant $\left(r_{p}=0.6867 ; r_{g}=\right.$ 0.8328 ), number of immature pods per plant $\left(r_{p}=0.1104 ; r_{g}=0.1434\right)$, shelling per cent $\left(r_{p}\right.$ $\left.=0.0362 ; r_{\mathrm{g}}=0.1009\right)$, SPAD chlorophyll meter reading $\left(r_{p}=0.0369 ; r_{g}=0.1650\right)$ and pod yield per plant $\left(r_{p}=0.1855 ; r_{g}=0.2203\right)$. Number of secondary branches per plant registered positive correlation with number of well-filled and mature pods per plant $\left(\mathrm{r}_{\mathrm{p}}=\right.$ $\left.0.0422 ; r_{\mathrm{g}}=0.0658\right)$, number of immature pods per plant $\left(r_{p}=0.0742 ; r_{g}=0.0908\right)$, dry haulms 
yield per plant $\left(r_{p}=0.0030 ; r_{g}=0.1805\right)$, shelling per cent $\left(r_{p}=0.0724 ; r_{g}=0.1020\right)$, SPAD chlorophyll meter reading $\left(\mathrm{r}_{\mathrm{p}}=0.1471\right.$; $\left.r_{\mathrm{g}}=0.2287\right)$ and pod yield per plant $\left(r_{p}=\right.$ $\left.0.0475 ; r_{g}=0.0437\right)$. Number of well-filled and mature pods per plant had shown positive correlation with SPAD Chlorophyll meter reading $\left(\mathrm{r}_{\mathrm{p}}=0.2334\right)$ at phenotypic level. Number of immature pods per plant shown negatively significant for 100 pod weight $\left(r_{p}=\right.$ -0.4970) and Specific leaf area $\left(r_{p}=-0.6813\right)$ at phenotypic level. Dry haulms yield per plant exhibited 100 pod weight $\left(r_{p}=0.0134\right.$; $\left.r_{g}=0.4794\right)$ and 100 kernel weight $\left(r_{p}=\right.$ 0.0644; $\left.r_{g}=1.0787\right)$. Similarly significant positive correlation of dry haulms yield per plant, 100-kernel weight with pod yield per plant are in accordance with the reports of Kahate et al., (2014), Ram et al., (2017), Hampannavar et al., (2018), Rao et al., (2014), John and Raghava Reddy (2019).

100- pod weight had shown positive significant correlation with sound mature kernel per cent $\left(r_{p}=0.1823\right)$, specific leaf area $\left(r_{p}=0.3585\right)$ and SPAD chlorophyll meter reading $\left(r_{p}=0.2715^{* *}\right)$ at phenotypic level. Shelling per cent registered positive correlation with 100 kernel weight $\left(r_{p}=\right.$ $\left.0.0550 ; r_{g}=0.0428\right)$, specific leaf area $\left(r_{p}=\right.$ $\left.0.0899 ; r_{g}=0.1414\right)$ and pod yield per plant $\left(r_{p}\right.$ $=0.6867 ; r_{\mathrm{g}}=0.8328$ ). 100- kernel weight had shown positive correlation with sound mature kernel per cent $\left(r_{p}=0.0437 ; r_{g}=0.6150\right)$ and SPAD chlorophyll meter reading $\left(\mathrm{r}_{\mathrm{p}}=\right.$ $\left.0.2118^{*} ; r_{g}=0.4218\right)$. Sound mature kernel per cent exhibited positive correlation with SPAD chlorophyll meter reading $\left(r_{p}=0.1141 ; r_{g}=\right.$ 0.5918 ) and negative correlation with specific leaf area $\left(r_{p}=-0.1292 ; r_{g}=-0.0165\right)$ and SPAD chlorophyll meter $\left(r_{p}=0.0027 ; r_{g}=-0.0923\right)$.

Specific leaf area exhibited negative correlation with SPAD chlorophyll meter reading $\left(\mathrm{r}_{\mathrm{p}}=-0.1979 *\right)$ at phenotypic level and pod yield per plant at genotypic level $\left(r_{g}=\right.$ -
0.4090). SPAD chlorophyll meter reading registered negative significant correlation with pod yield per plant $\left(r_{g}=-0.3150\right)$. Pod yield per plant showed positively significant correlation with the traits viz., number of primary branches per plant $\left(r_{\mathrm{g}}=0.2203\right)$, number of immature pods per plant $\left(\mathrm{r}_{\mathrm{p}}=\right.$ $\left.0.2548 ; r_{g}=0.3606\right)$ and 100 pod weight $\left(r_{g}=0.3030\right)$. Significant positive correlation of pod yield per plant with number of primary branches per plant was reported by Korat et al., (2009), Khanpara et al., (2010), Bhavya et al., (2017).

\section{Path analysis}

To know the direct and indirect effects of seed yield and yield related traits correlation coefficient was further partitioned into direct and indirect effects through path coefficient analysis by considering pod yield as a dependent character. Yield is the sum total of the several component characters which directly or indirectly contributed to it. The information derived from the correlation studies indicated only mutual association among the characters. Whereas, path coefficient analysis helps in understanding the nature of magnitude of direct and indirect contribution of each character on the dependent character like seed yield per plant. The results of phenotypic and genotypic path coefficients were presented in Table 2.

The highest positive direct effect on pod yield per plant was recorded with number of primary branches per plant $\left(\mathrm{P}_{\mathrm{g}}=1.4667\right)$ followed by sound mature kernel per cent $\left(\mathrm{P}_{\mathrm{g}}=\right.$ 1.4615), 100 kernel weight $\left(\mathrm{P}_{\mathrm{g}}=0.7693\right)$ and number of well-filled and mature pods per plant $\left(\mathrm{P}_{\mathrm{g}}=0.3488\right)$ at genotypic level.

Conversely, negative and negligible direct effect on pod yield per plant by plant height $\left(P_{g}=-0.5980\right)$, number of secondary branches per plant $\left(-\left(\mathrm{P}_{\mathrm{g}}=-1.1273\right)\right.$, number of immature 
pods per plant $\left(\left(\mathrm{P}_{\mathrm{g}}=-1.0946\right)\right.$, dry haulms yield per plant $\left(\mathrm{P}_{\mathrm{g}}=-0.0203\right), 100$ pod weight $\left(P_{g}=-0.3711\right)$, shelling per cent $\left(P_{g}=-0.0061\right)$, specific leaf area $\left(\mathrm{P}_{\mathrm{g}}=-0.3685\right)$ followed by SPAD chlorophyll meter reading $\left(\left(\mathrm{P}_{\mathrm{g}}=-\right.\right.$ 0.5231) at genotypic level.

Plant height exhibited the negative indirect effect of plant height on pod yield per plant via number of primary branches per plant $\left(\mathrm{P}_{\mathrm{p}}=\right.$ $-0.0122)$, dry haulms yield per plant $\left(\mathrm{P}_{\mathrm{p}}=-\right.$ $0.0069), 100$ pod weight $\left(\mathrm{P}_{\mathrm{p}}=-0.0149\right)$ and specific leaf area $\left(\mathrm{P}_{\mathrm{p}}=-0.0316\right)$ at phenotypic level and at genotypic level via number of secondary branches per plant $\left(\mathrm{P}_{\mathrm{g}}=0.0658\right)$, number of well-filled and mature pods per plant $\left(\mathrm{P}_{\mathrm{g}}=-0.5012\right)$, number of immature pods per plant $\left(\mathrm{P}_{\mathrm{g}}=-0.1487\right)$, dry haulms yield per plant $\left(\mathrm{P}_{\mathrm{g}}=-0.6041\right)$, shelling per cent $\left(\mathrm{P}_{\mathrm{g}}=\right.$ 0.0694), 100 kernel weight $\left(\mathrm{P}_{\mathrm{g}}=-0.1318\right)$, sound mature kernel per cent $\left(\mathrm{P}_{\mathrm{p}}=-0.1853\right.$ and SPAD chlorophyll meter reading $\left(\mathrm{P}_{\mathrm{p}}=\right.$ 0.1764 ) at phenotypic level.

Number of primary branches per plant had shown positive and negligible indirect effects on pod yield per plant through number of primary branches per plant $\left(\mathrm{P}_{\mathrm{p}}=0.2752\right.$;

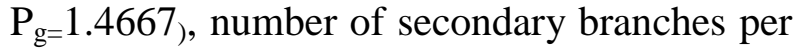
plant $\left(\mathrm{P}_{\mathrm{p}}=0.1890 ; \mathrm{P}_{\mathrm{g}=1.2215}\right)$, number of immature pods per plant $\left(\mathrm{P}_{\mathrm{p}}=0.0304\right.$; $\mathrm{P}_{\mathrm{g}=0.2104}$, shelling per cent $\left(\mathrm{P}_{\mathrm{p}}=0.0100\right.$;

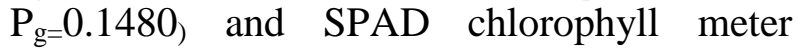
reading $\left(\mathrm{P}_{\mathrm{p}}=0.0101 ; \mathrm{P}_{\mathrm{g}=0.2420}\right.$ )

The negative indirect effect of number of primary branches per plant on pod yield per plant through plant height $\left(\mathrm{P}_{\mathrm{p}}=-0.0208 ; \mathrm{P}_{\mathrm{g}=-}\right.$ $0.161), 100$ pod weight $\left(\mathrm{P}_{\mathrm{p}}=-0.0216 ; \mathrm{P}_{\mathrm{g}=}=\right.$ $0.1687), 100$ kernel weight $\left(\mathrm{P}_{\mathrm{p}}=-0.0271 ; \mathrm{P}_{\mathrm{g}=}=^{-}\right.$ $0.2839)$, sound mature kernel per cent $\left(\mathrm{P}_{\mathrm{p}}=-\right.$ $0.0186 ; \mathrm{P}_{\mathrm{g}}=-0.2728$, and specific leaf area.

Number of secondary branches per plant exhibited positive and negligible indirect effect on pod yield per plant via 100 pod weight $\left(\mathrm{P}_{\mathrm{p}}=0.0011 ; \mathrm{P}_{\mathrm{g}=-0}\right.$. 1012) and 100 kernel weight $\left(\mathrm{P}_{\mathrm{p}}=0.0088 ; \mathrm{P}_{\mathrm{g}=0} 0\right.$. 0919) and

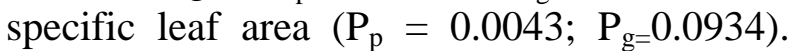
Number of well-filled and mature pod per plant had shown positive and negligible indirect effect on pod yield per plant via dry haulms yield per plant $\left(\mathrm{P}_{\mathrm{p}}=0.0005 ; \mathrm{P}_{\mathrm{g}}\right.$ $=0.5098)$. Negative and negligible indirect effects through number of primary branches per plant $\left(P_{p}=-0.0004 ; P_{g}=-0.0438\right)$. Number of immature pods per plant exhibited negative negligible indirect effect on pod yield per plant via dry haulms yield per plant $\left(\mathrm{P}_{\mathrm{p}}=\right.$ -

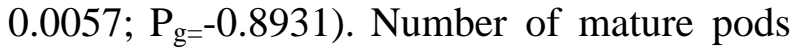
per plant exhibited positive direct effect and positive association at both genotypic and phenotypic level which were confirmed with the findings of Suneetha et al., (2005), Venkateswarulu et al., (2007), Parameshwarappa et al., (2008), Zaman et al., (2011), Vaithiyalingam et al., (2010) and Bhargavi et al., (2017).

Dry haulms yield per plant had shown positive and negligible indirect effects on pod yield per plant via sound mature kernel per cent $\left(\mathrm{P}_{\mathrm{p}}=\right.$ 0.0013; $\mathrm{P}_{\mathrm{g}}=0.0261$ ) and negative negligible indirect effects through plant height $\left(\mathrm{P}_{\mathrm{p}}=\right.$ -

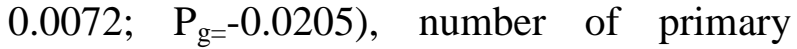
branches per plant $\left(\mathrm{P}_{\mathrm{p}}=-0.0054 ; \mathrm{P}_{\mathrm{g}=-0.0127}\right)$ and number of immature pods per plant $\left(\mathrm{P}_{\mathrm{p}}=-\right.$

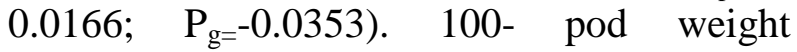
exhibited negative and negligible indirect effect on pod yield per plant via plant height $\left(\mathrm{P}_{\mathrm{p}}=-0.0066\right)$, number of primary branches per plant $\left(\mathrm{P}_{\mathrm{p}}=-0.0056\right)$ and number of secondary branches per plant $\left(\mathrm{P}_{\mathrm{p}}=-0.0005\right)$ at phenotypic level. Conversely, number of wellfilled and mature pods per plant $\left(\mathrm{P}_{\mathrm{g}}=-1.0678\right)$, dry haulms yield per plant $\left(\mathrm{P}_{\mathrm{g}}=-1.1367\right), 100$ pod weight $\left(\mathrm{P}_{\mathrm{g}}=-2.3711\right)$, shelling per cent $\left(\mathrm{P}_{\mathrm{g}}=\right.$-0.0636), 100 kernel weight $\left(\mathrm{P}_{\mathrm{g}}=\right.$ 1.3298), sound mature kernel per cent $\left(\mathrm{P}_{\mathrm{g}}=\right.$ 1.8988), specific leaf area $\left(\mathrm{P}_{\mathrm{g}}=-0.8923\right)$ and SPAD chlorophyll meter reading $\left(\mathrm{P}_{\mathrm{g}}=\right.$ 1.2820). 
Shelling per cent shown positive and negligible indirect effect on pod yield per plant via plant height $\left(\mathrm{P}_{\mathrm{p}}=0.0045\right.$;

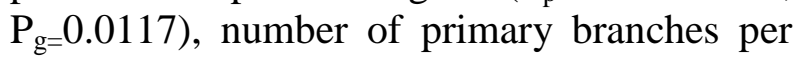
plant $\left(\mathrm{P}_{\mathrm{p}}=0.0018 ; \mathrm{P}_{\mathrm{g}=0.0101)}\right.$ number of secondary branches per plant $\left(\mathrm{P}_{\mathrm{p}}=0.0035\right.$; $\mathrm{P}_{\mathrm{g}=0.0102)}$ number of well-filled and mature pods per plant $\left(\mathrm{P}_{\mathrm{p}}=0.0049 ; \mathrm{P}_{\mathrm{g}=0.0051), 100}\right.$

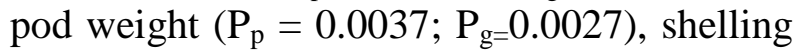

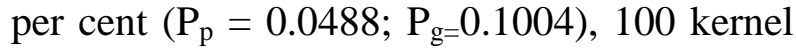
weight $\left(\mathrm{P}_{\mathrm{p}}=0.0027 ; \mathrm{P}_{\mathrm{g}=0.0043}\right)$ and specific leaf area $\left(\mathrm{P}_{\mathrm{p}}=0.0044 ; \mathrm{P}_{\mathrm{g}=0.0142}\right)$. Similar findings were also reported by Azad and Hamid (2000), Siddiquey et al., (2006), Parameswarappa et al., (2008) and Vaithiyalingan et al., (2010).

100- kernel weight exhibited positive and negligible indirect effect on pod yield per plant via number of primary branches per plant $\left(\mathrm{P}_{\mathrm{p}}=0.0109\right)$ number of secondary branches per plant $\left(\mathrm{P}_{\mathrm{p}}=0.0069\right)$ and specific leaf area $\left(\mathrm{P}_{\mathrm{p}}=0.0081\right)$ at phenotypic level. In contrast, negative and negligible indirect effects on pod yield per plant was noted by plant height $\left(\mathrm{P}_{\mathrm{p}}=-0.0137\right)$, number of wellfilled and mature pods per plant $\left(\mathrm{P}_{\mathrm{p}}=-0.0159\right)$, number of immature pods per plant $\left(\mathrm{P}_{\mathrm{p}}=\right.$ $0.0150)$, dry haulms yield per plant $\left(\mathrm{P}_{\mathrm{p}}=\right.$ 0.0071), 100 pod weight $\left(\mathrm{P}_{\mathrm{p}}=-0.0378\right)$, shelling per cent $\left(\mathrm{P}_{\mathrm{p}}=-0.0061\right), 100$ kernel weight $\left(P_{p}=-0.1108\right)$, sound mature kernel per cent $\left(\mathrm{P}_{\mathrm{p}}=-0.0048\right)$ and SPAD chlorophyll meter reading $\left(\mathrm{P}_{\mathrm{p}}=-0.0235\right)$ at phenotypic level and number of primary branches per plant $\left(\mathrm{P}_{\mathrm{g}}=-0.1489\right)$ and number of secondary branches per plant $\left(\mathrm{P}_{\mathrm{g}}=-0.0627\right)$ at genotypic level.

Sound mature kernel per cent shown positive and negligible indirect effect on pod yield per plant via plant height $\left(\mathrm{P}_{\mathrm{p}}=0.0007\right.$;

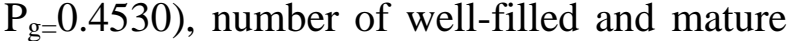
pods per plant $\left(\mathrm{P}_{\mathrm{p}}=0.0002 ; \mathrm{P}_{\mathrm{g}=1.0074}\right), 100$ pod weight $\left(\mathrm{P}_{\mathrm{p}}=0.0008 ; \mathrm{P}_{\mathrm{g}}=1.1704\right), 100$ kernel weight $\left(\mathrm{P}_{\mathrm{p}}=0.7693 ; \mathrm{P}_{\mathrm{g}=0.0002}\right)$, sound mature kernel per cent $\left(\mathrm{P}_{\mathrm{p}}=0.0044\right.$; $\mathrm{P}_{\mathrm{g}=1.4615)}$ and SPAD chlorophyll meter reading $\left(P_{p}=0.0005 ; P_{g=} 0.8693\right)$.

Specific leaf area exhibited positive and negligible indirect effect on pod yield per plant via plant height $\left(\mathrm{P}_{\mathrm{p}}=0.0241\right.$; $\mathrm{P}_{\mathrm{g}=0.1230}$ ), number of primary branches per plant $\left(\mathrm{P}_{\mathrm{p}}=0.0069 ; \mathrm{P}_{\mathrm{g}=0.0644}\right)$, number of secondary branches per plant $\left(\mathrm{P}_{\mathrm{p}}=0.0038\right.$;

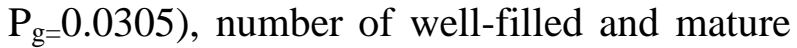
pods per plant $\left(\mathrm{P}_{\mathrm{p}}=0.0097 ; \mathrm{P}_{\mathrm{g}=0.1982}\right)$, number of immature pods per plant $\left(\mathrm{P}_{\mathrm{p}}=\right.$

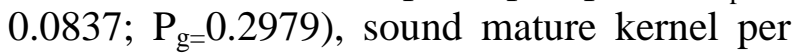
cent $\left(\mathrm{P}_{\mathrm{p}}=0.0159 ; \mathrm{P}_{\mathrm{g}=0.0061}\right)$ and SPAD chlorophyll meter reading $\left(\mathrm{P}_{\mathrm{p}}=0.0243\right.$;

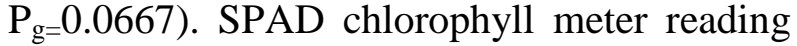
had shown positive and negligible indirect effect on pod yield per plant via number of immature pods per plant $\left(\mathrm{P}_{\mathrm{p}}=0.0064\right.$;

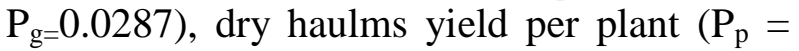
$0.0023 ; \mathrm{P}_{\mathrm{g}=0.3840)}$ and specific leaf area $\left(\mathrm{P}_{\mathrm{p}}=\right.$

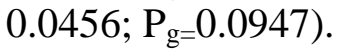

Pod yield per plant showed positively significant correlation with the traits viz., number of primary branches per plant, number of immature pods per plant and 100 pod weight. These characters can be considered as criteria for selection for higher yield, as these were mutually and directly associated with pod yield. The highest positive direct effect on pod yield per plant was recorded with number of primary branches per plant followed by sound mature kernel per cent, 100 kernel weight and number of well-filled and mature pods per plant. Hence, a direct selection for this trait would be effective. 
Table.1 Phenotypic (P) and genotypic (G) correlation coefficients among yield and yield traits in groundnut

\begin{tabular}{|c|c|c|c|c|c|c|c|c|c|c|c|c|c|c|}
\hline Character & $\mathbf{r}$ & $\begin{array}{c}\text { Plant } \\
\text { height }\end{array}$ & $\begin{array}{c}\text { Number } \\
\text { of } \\
\text { primary } \\
\text { branches } \\
\text { per plant }\end{array}$ & $\begin{array}{l}\text { Number of } \\
\text { secondary } \\
\text { branches } \\
\text { per plant }\end{array}$ & $\begin{array}{c}\text { Number of } \\
\text { well-filled } \\
\text { and } \\
\text { mature } \\
\text { pods per } \\
\text { plant }\end{array}$ & $\begin{array}{c}\text { Number of } \\
\text { immature } \\
\text { pods per } \\
\text { plant }\end{array}$ & $\begin{array}{c}\text { Dry } \\
\text { haulm } \\
\text { s yield } \\
\text { per } \\
\text { plant } \\
(\mathrm{g})\end{array}$ & $\begin{array}{c}100 \text { pod } \\
\text { weight }(g)\end{array}$ & $\begin{array}{l}\text { Shelling } \\
\text { per cent }\end{array}$ & $\begin{array}{c}100 \\
\text { kernel } \\
\text { weight }(\mathrm{g})\end{array}$ & $\begin{array}{c}\text { Sound } \\
\text { mature } \\
\text { kernel } \\
\text { per cent }\end{array}$ & $\begin{array}{r}\text { Specific } \\
\text { leaf area }\end{array}$ & $\begin{array}{c}\text { SAPD } \\
\text { Chloroph } \\
\text { yll meter } \\
\text { reading }\end{array}$ & $\begin{array}{l}\text { Pod yield } \\
\text { per plant }\end{array}$ \\
\hline \multirow[t]{2}{*}{ Plant height } & $r_{p}$ & 1.0000 & -0.0756 & 0.0504 & $\begin{array}{c}0.2263 * \\
*\end{array}$ & $0.1882 *$ & $\begin{array}{c}- \\
0.0429\end{array}$ & -0.0926 & 0.0921 & 0.1236 & 0.1526 & $-0.1965^{*}$ & $0.2041 *$ & 0.0107 \\
\hline & $r_{g}$ & 1.0000 & -0.1100 & 0.0546 & 0.8382 & 0.2487 & 1.0102 & -0.1470 & 0.1161 & 0.2204 & 0.3099 & -0.3339 & 0.2950 & 0.1301 \\
\hline \multirow[t]{2}{*}{$\begin{array}{c}\text { Number of primary branches } \\
\text { per plant }\end{array}$} & $r_{p}$ & & 1.0000 & $0.6867 * *$ & 0.0062 & 0.1104 & 0.0323 & -0.0783 & 0.0362 & -0.0986 & -0.0675 & -0.0565 & 0.0369 & 0.1855 \\
\hline & $r_{g}$ & & 1.0000 & 0.8328 & -0.1257 & 0.1434 & 0.6250 & -0.1150 & 0.1009 & -0.1936 & -0.1860 & -0.1747 & 0.1650 & 0.2203 \\
\hline \multirow{2}{*}{$\begin{array}{c}\text { Number of secondary branches } \\
\text { per plant }\end{array}$} & $r_{p}$ & & & 1.0000 & 0.0422 & 0.0742 & 0.0030 & -0.0077 & 0.0724 & -0.0624 & 0.0526 & -0.0307 & 0.1471 & 0.0475 \\
\hline & $r_{g}$ & & & 1.0000 & 0.0658 & 0.0908 & 0.1805 & -0.0898 & 0.1020 & -0.0815 & -0.0063 & -0.0828 & 0.2287 & 0.0437 \\
\hline \multirow[t]{2}{*}{$\begin{array}{l}\text { Number of well-filled and } \\
\text { mature pods per plant }\end{array}$} & $r_{p}$ & & & & 1.0000 & 0.0302 & $\begin{array}{c}- \\
0.0086\end{array}$ & 0.0955 & 0.0997 & 0.1437 & 0.0438 & -0.0787 & $0.2334 * *$ & -0.0760 \\
\hline & $r_{g}$ & & & & 1.0000 & 0.1429 & 1.4616 & 0.4503 & 0.0510 & 0.9257 & 0.6893 & -0.5378 & 0.9793 & -0.2543 \\
\hline \multirow[t]{2}{*}{$\begin{array}{c}\text { Number of immature pods per } \\
\text { plant }\end{array}$} & $r_{p}$ & & & & & 1.0000 & 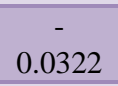 & $-0.4970 * *$ & -0.0910 & 0.1350 & -0.0160 & $-0.6813 * *$ & -0.0276 & 0.2548 \\
\hline & $r_{g}$ & & & & & 1.0000 & 0.8159 & -0.6552 & -0.1281 & 0.1072 & -0.3333 & -0.8084 & -0.0548 & 0.3606 \\
\hline \multirow[t]{2}{*}{ Dry haulms yield per plant (g) } & $r_{p}$ & & & & & & 1.0000 & 0.0134 & -0.0223 & 0.0644 & 0.0614 & -0.0165 & -0.0101 & 0.1436 \\
\hline & $r_{g}$ & & & & & & 1.0000 & 0.4794 & 1.3229 & 1.0787 & -1.2843 & 0.6777 & -0.7341 & -2.2111 \\
\hline \multirow[t]{2}{*}{100 pod weight (g) } & $r_{p}$ & & & & & & & 1.0000 & 0.0758 & $0.3416 * *$ & $0.1823^{* *}$ & $0.3585 * *$ & $0.2715^{* *}$ & -0.1960 \\
\hline & $r_{g}$ & & & & & & & 1.0000 & 0.0268 & 0.5609 & 0.8008 & 0.3763 & 0.5407 & 0.3030 \\
\hline \multirow[t]{2}{*}{ Shelling per cent } & $r_{p}$ & & & & & & & & 1.0000 & 0.0550 & -0.0545 & 0.0899 & -0.0041 & 0.0261 \\
\hline & $r_{g}$ & & & & & & & & 1.0000 & 0.0428 & -0.0328 & 0.1414 & 0.0528 & 0.0369 \\
\hline \multirow[t]{2}{*}{100 kernel weight (g) } & $r_{p}$ & & & & & & & & & 1.0000 & 0.0437 & -0.0734 & $0.2118^{*}$ & -0.0965 \\
\hline & $\mathrm{r}_{\mathrm{g}}$ & & & & & & & & & 1.0000 & 0.6150 & 0.0607 & 0.4218 & -0.0406 \\
\hline \multirow[t]{2}{*}{ Sound mature kernel per cent } & $r_{p}$ & & & & & & & & & & 1.0000 & -0.1292 & 0.1141 & 0.0027 \\
\hline & $r_{g}$ & & & & & & & & & & 1.0000 & -0.0165 & 0.5948 & -0.0923 \\
\hline \multirow[t]{2}{*}{ Specific leaf area } & $r_{p}$ & & & & & & & & & & & 1.0000 & $-0.1979 *$ & -0.2008 \\
\hline & $r_{g}$ & & & & & & & & & & & 1.0000 & -0.1810 & -0.4090 \\
\hline \multirow{2}{*}{$\begin{array}{l}\text { SAPD Chlorophyll meter } \\
\text { reading }\end{array}$} & $r_{p}$ & & & & & & & & & & & & 1.0000 & -0.2091 \\
\hline & $r_{g}$ & & & & & & & & & & & & 1.0000 & -0.3150 \\
\hline
\end{tabular}

$*$ Significant at $5 \%$ level $* *$ Significant at $1 \%$ level 
Table.2 Path coefficients for yield and yield traits in groundnut

\begin{tabular}{|c|c|c|c|c|c|c|c|c|c|c|c|c|c|}
\hline Character & & $\begin{array}{c}\text { Plant } \\
\text { height }\end{array}$ & $\begin{array}{c}\begin{array}{c}\text { Number } \\
\text { of } \\
\text { primary }\end{array} \\
\text { branches } \\
\text { per plant }\end{array}$ & $\begin{array}{l}\text { Number of } \\
\text { secondary } \\
\text { branches } \\
\text { per plant }\end{array}$ & $\begin{array}{l}\text { Number of } \\
\text { well-filled } \\
\text { and mature } \\
\text { pods per } \\
\text { plant }\end{array}$ & $\begin{array}{c}\text { Number of } \\
\text { immature } \\
\text { pods per } \\
\text { plant }\end{array}$ & $\begin{array}{c}\text { Dry } \\
\text { haulms } \\
\text { yield per } \\
\text { plant }(\mathrm{g})\end{array}$ & $\begin{array}{c}100 \text { pod } \\
\text { weight }(\mathrm{g})\end{array}$ & $\begin{array}{l}\text { Shelling } \\
\text { per cent }\end{array}$ & $\begin{array}{c}100 \\
\text { kernel } \\
\text { weight }(g)\end{array}$ & $\begin{array}{c}\text { Sound } \\
\text { mature } \\
\text { kernel } \\
\text { per cent }\end{array}$ & $\begin{array}{r}\text { Specific } \\
\text { leaf area }\end{array}$ & $\begin{array}{l}\text { SAPD } \\
\text { Chlorophyl } \\
\text { I meter } \\
\text { reading }\end{array}$ \\
\hline \multirow[t]{2}{*}{ Plant height } & $\mathrm{Pp}$ & 0.1611 & -0.0122 & 0.0081 & 0.0365 & 0.0303 & -0.0069 & -0.0149 & 0.0148 & 0.0199 & 0.0246 & -0.0316 & 0.0329 \\
\hline & $\mathrm{Pg}$ & -0.5980 & 0.0658 & -0.0327 & -0.5012 & -0.1487 & -0.6041 & 0.0879 & -0.0694 & -0.1318 & -0.1853 & 0.1996 & -0.1764 \\
\hline \multirow{2}{*}{$\begin{array}{l}\text { Number of primary } \\
\text { branches per plant }\end{array}$} & $\mathrm{Pp}$ & -0.0208 & 0.2752 & 0.1890 & 0.0017 & 0.0304 & -0.0089 & -0.0216 & 0.0100 & -0.0271 & -0.0186 & -0.0155 & 0.0101 \\
\hline & $\mathrm{Pg}$ & -0.1614 & 1.4667 & 1.2215 & -0.1844 & 0.2104 & 0.9168 & -0.1687 & 0.1480 & -0.2839 & -0.2728 & -0.2562 & 0.2420 \\
\hline \multirow{2}{*}{$\begin{array}{l}\text { Number of secondary } \\
\text { branches per plant }\end{array}$} & $\mathrm{Pp}$ & -0.0071 & -0.0965 & -0.1406 & -0.0059 & -0.0104 & -0.0004 & 0.0011 & -0.0102 & 0.0088 & -0.0074 & 0.0043 & -0.0207 \\
\hline & $\mathrm{Pg}$ & -0.0616 & -0.9389 & -1.1273 & -0.0742 & -0.1023 & -0.2035 & 0.1012 & -0.1150 & 0.0919 & 0.0071 & 0.0934 & -0.2578 \\
\hline \multirow{2}{*}{$\begin{array}{c}\text { Number of well-filled } \\
\text { and mature pods per } \\
\text { plant }\end{array}$} & $\mathrm{Pp}$ & -0.0145 & -0.0004 & -0.0027 & -0.0640 & -0.0019 & 0.0005 & -0.0061 & -0.0064 & -0.0092 & -0.0028 & 0.0050 & -0.0149 \\
\hline & $\mathrm{Pg}$ & 0.2924 & -0.0438 & 0.0229 & 0.3488 & 0.0498 & 0.5098 & 0.1571 & 0.0178 & 0.3229 & 0.2404 & -0.1876 & 0.3416 \\
\hline \multirow{2}{*}{$\begin{array}{l}\text { Number of immature } \\
\text { pods per plant }\end{array}$} & $\mathrm{Pp}$ & 0.0332 & 0.0195 & 0.0131 & 0.0053 & 0.1766 & -0.0057 & -0.0878 & -0.0161 & 0.0238 & -0.0028 & -0.1203 & -0.0049 \\
\hline & $\mathrm{Pg}$ & -0.2723 & -0.1570 & -0.0994 & -0.1564 & -1.0946 & -0.8931 & 0.7172 & 0.1402 & -0.1173 & 0.3648 & 0.8849 & 0.0600 \\
\hline \multirow{2}{*}{$\begin{array}{c}\text { Dry haulms yield per } \\
\text { plant (g) }\end{array}$} & $\mathrm{Pp}$ & -0.0072 & -0.0054 & 0.0005 & -0.0014 & -0.0054 & 0.1676 & 0.0022 & -0.0037 & 0.0108 & 0.0103 & -0.0028 & -0.0017 \\
\hline & $\mathrm{Pg}$ & -0.0205 & -0.0127 & -0.0037 & -0.0297 & -0.0166 & -0.0203 & -0.0097 & -0.0269 & -0.0219 & 0.0261 & -0.0138 & 0.0149 \\
\hline \multirow[t]{2}{*}{100 pod weight (g) } & $\mathrm{Pp}$ & -0.0066 & -0.0056 & -0.0005 & 0.0068 & -0.0353 & 0.0010 & 0.0709 & 0.0054 & 0.0242 & 0.0129 & 0.0254 & 0.0193 \\
\hline & $\mathrm{Pg}$ & 0.3485 & 0.2727 & 0.2129 & -1.0678 & 1.5536 & -1.1367 & -2.3711 & -0.0636 & -1.3298 & -1.8988 & -0.8923 & -1.2820 \\
\hline \multirow[t]{2}{*}{ Shelling per cent } & $\mathrm{Pp}$ & 0.0045 & 0.0018 & 0.0035 & 0.0049 & -0.0044 & -0.0011 & 0.0037 & 0.0488 & 0.0027 & -0.0027 & 0.0044 & -0.0002 \\
\hline & $\mathrm{Pg}$ & 0.0117 & 0.0101 & 0.0102 & 0.0051 & -0.0129 & 0.1328 & 0.0027 & 0.1004 & 0.0043 & -0.0033 & 0.0142 & 0.0053 \\
\hline \multirow[t]{2}{*}{100 kernel weight (g) } & $\mathrm{Pp}$ & -0.0137 & 0.0109 & 0.0069 & -0.0159 & -0.0150 & -0.0071 & -0.0378 & -0.0061 & -0.1108 & -0.0048 & 0.0081 & -0.0235 \\
\hline & $\mathrm{Pg}$ & 0.1695 & -0.1489 & -0.0627 & 0.7121 & 0.0824 & 0.8298 & 0.4315 & 0.0329 & 0.7693 & 0.4731 & 0.0467 & 0.3245 \\
\hline \multirow{2}{*}{$\begin{array}{l}\text { Sound mature kernel } \\
\text { per cent }\end{array}$} & $\mathrm{Pp}$ & 0.0007 & -0.0003 & 0.0002 & 0.0002 & -0.0001 & 0.0003 & 0.0008 & -0.0002 & 0.0002 & 0.0044 & -0.0006 & 0.0005 \\
\hline & $\mathrm{Pg}$ & 0.4530 & -0.2719 & -0.0091 & 1.0074 & -0.4871 & -1.8770 & 1.1704 & -0.0479 & 0.8988 & 1.4615 & -0.0241 & 0.8693 \\
\hline \multirow[t]{2}{*}{ Specific leaf area } & $\mathrm{Pp}$ & 0.0241 & 0.0069 & 0.0038 & 0.0097 & 0.0837 & 0.0020 & -0.0440 & -0.0110 & 0.0090 & 0.0159 & -0.1228 & 0.0243 \\
\hline & $\mathrm{Pg}$ & 0.1230 & 0.0644 & 0.0305 & 0.1982 & 0.2979 & -0.2497 & -0.1387 & -0.0521 & -0.0224 & 0.0061 & -0.3685 & 0.0667 \\
\hline \multirow{2}{*}{$\begin{array}{l}\text { SAPD Chlorophyll } \\
\text { meter reading }\end{array}$} & $\mathrm{Pp}$ & -0.0470 & -0.0085 & -0.0339 & -0.0538 & 0.0064 & 0.0023 & -0.0626 & 0.0009 & -0.0488 & -0.0263 & 0.0456 & -0.2304 \\
\hline & $\mathrm{Pg}$ & -0.1543 & -0.0863 & -0.1196 & -0.5122 & 0.0287 & 0.3840 & -0.2828 & -0.0276 & -0.2207 & -0.3111 & 0.0947 & -0.5231 \\
\hline \multirow[t]{2}{*}{ Pod yield } & $\mathrm{Pp}$ & 0.1068 & 0.1855 & 0.0475 & -0.0760 & 0.2548 & 0.1436 & -0.1960 & 0.0261 & -0.0965 & 0.0027 & -0.2008 & -0.2091 \\
\hline & $\mathrm{Pg}$ & 0.1301 & 0.2203 & 0.0437 & -0.2543 & 0.3606 & -2.2111 & -0.3030 & 0.0369 & -0.0406 & -0.0923 & -0.4090 & -0.3150 \\
\hline
\end{tabular}

Diagonal values (Bold) : Direct effects 


\section{Acknowledgement}

This research was supported under the DSTSERB to the first author by the Department of Science and Technology -Science and Engineering Research Board, New Delhi is gratefully acknowledged.

\section{References}

Al-Jibouri, H. A., Miller, P. A. and Robinson, H. F., (1958). Genotypic and environmental variance and covariance in an upland cotton cross of interspecific origin. Agronomy J. 50: 633637.

Azad, M. A. K. And Hamid, M. A., (2000). Genetic variability, character association and path analysis in groundnut (Arachis hypogaea L.). Thailand J. of Agric. Sci. 33: 153-157.

Bhargavi, G., Rao, S.V., Babu, D.R and Rao, N.K.L. 2017. Character association and path coefficient analysis of pod yield and yield components in Virginia bunch groundnut (Arachis hypogaea L.). Electronic Journal of Plant Breeding. 8(1): 262-268.

Bhavya, M.R., Shanthala, J., Savithramma, D.L and Sab, S. 2017. Variability, heritability and association studies in $F_{4}$ and $F_{5}$ generation for traits related to water use efficiency and yield traits in groundnut (Arachis hypogea L.). Plant Archives. 17(2): 1353-1360.

Dewey, D. R. and Lu, K. H., (1959). A correlation and path analysis of components of crested wheat grass seed production. Agronomy J., 5: 515518.

Hampannavar, M.R., Khan, H., Temburne, B.V., Janila, P and Amaregouda, A. 2018. Genetic variability, correlation and path analysis studies for yield and yield attributes in groundnut
(Arachis hypogaea L.). Journal of Pharmacognosy and Phytochemistry. 7(1): 870-874.

John and Raghava Reddy (2019). Correlation and path analysis for yield and yield attributes in groundnut (Arachis hypogaea L.). Legume Research, 42(4) 2019: 518-522

Kahate, N.S., Toprope, V.N and Gadakh, S.S. 2014. Correlation and path analysis for yield, morphology and biochemical traits in groundnut (Arachis hypogea L.). Bioinfolet. 11(3B): 868-870.

Khanpara, M.D., Shinde, P.P., Jivani, L.L., Vacchani, J.H and Kachhadia, V.H. 2010. Character association and path coefficient analysis in groundnut (Arachis hypogea L.). Plant Archives. 10(2): 695-698.

Korat, V.P., Pithia, M.S., Savaliya, J.J., Pansuriya. A.G and Sodavadiya, P.R. 2009. Studies on genetic variability in different genotypes of groundnut (Arachis hypogaea L.). Legume Research. 33(2): 224-226.

Mahalakshmi, P., Manivannan, N. and Muralidharan, V., (2005). Variability and correlation studies in groundnut (Arachis hypogaea L.). Legume Res. 28 (3):194-197.

Parameshwarappa, K. G., Malabaari, T. A. and Lingaraju, B. S., (2008). Analysis of correlation and path effects among yield attributing traits in two crosses of large seeded groundnut, Arachis hypogaea L. J. Oilsseds Res. 25 (10): 4-7.

Ram, D.T., Hemalata, S., Ramesh, K and Ram, K. 2017. Correlation and path analysis for yield and its contributing traits in groundnut (Arachis hypogaea L.). International Journal of Agriculture Sciences. 9 (10): 39973999.

Rao, V.T., Venkanna, V., Bhadru, D and 
Bharathi, D. 2014. Studies on variability, character association and path analysis on groundnut (Arachis hypogaea L.). International Journal of Pure and Applied Bioscience. 2 (2): 194-197.

Siddiquey, M. N., Haque, M. M., Ara, M. J. F., Ahmed, M. R. and Roknuzzaman, M., (2006). Correlation and Path analysis of groundnut (Arachis hypogaea L.). Int. J. of Sustainable Agric. Technology. 2 (7): 22-27.

Suneetha, K., Reddy, C.D.R and Ramana, J.V. 2005. Genetic variability and character association in groundnut (Arachis hypogaea L.). The Andhra Agricultural Journal. 52(1\&2): 43-47.

Venkateswarlu, O., Raja Reddy, K., Reddy, P. V., Vasanthi, R. P., Hariprasad Reddy,
K. and Eswara Reddy, N. P., (2007). Character association and path analysis for morphophysiological traits in groundnut Arachis hypogaea L. J. Oilseeds Res. 24 (1): 20-22.

Vaithiyalingam, M., Manoharan, V. and Ramamoorthi, N., (2010). Association analysisamong the yield attributes of early season drought tolerant groundnut (Arachis hypogaea L.). Electronic J. of Plant Breeding. 1(5): 1347-1350.

Zaman, M.A., Khatun, M.T., Ullah, M.Z., Moniruzzamn, $\mathrm{M}$ and Alam, K.H. 2011. Genetic variability and path analysis of groundnut (Arachis hypogaea L.). The Agriculturists. 9 (1\&2): 29-36.

\section{How to cite this article:}

John, K., M. V. Madhavi Santhoshi and Rajasekhar, P. 2019. Correlation and Path Analysis in Groundnut (Arachis hypogaea L.). Int.J.Curr.Microbiol.App.Sci. 8(12): 1521-1529. doi: https://doi.org/10.20546/ijcmas.2019.812.182 